\title{
Ultrasonic Studies of Ternary Mixtures of $N$-Alkanols in Ethylmethacrylate and Benzene at Various Temperatures
}

\author{
S. MEENA ${ }^{1}$, K. RAJU ${ }^{2 *}$ and A. N. KANNAPPAN ${ }^{3}$ \\ ${ }^{1}$ Department of Physics, M. R. Govt. Arts College, Mannargudi, Tamilnadu, India \\ ${ }^{2}$ Department of Physics, Sri Venkateswara College of Engineering, \\ Sriperumbudur-602105, India \\ ${ }^{3}$ Department of Physics, K.R College of Arts and Science, Kovilpatti, India \\ raju@svce.ac.in
}

Received 22 May 2014 / Accepted 2 June 2014

\begin{abstract}
Ultrasonic velocity, density and viscosity measurements have been measured for the ternary mixtures of 1-propanol and 1-butanol in ethyl methacrylate + benzene at 303,308 and $313 \mathrm{~K}$. From the experimental data adiabatic compressibility, free length, free volume internal pressure and their excess values have been calculated. These parameters are used to explain the nature of interactions between the component molecules of the mixtures.
\end{abstract}

Keywords: Ultrasonic velocity, Compressibility, n-Alkanols, Free length, Excess parameters

\section{Introduction}

During the last few decades, the measurement of ultrasonic velocity has been effectively used in understanding the nature of molecular interactions in liquid and liquid mixtures. Though the molecular interactions studies can be carried out by several spectroscopic techniques, the other non spectroscopic techniques such as dielectric, magnetic, viscosity and ultrasonic velocity measurements have been adequately used in the field of interactions and structural aspect evaluation studies ${ }^{1,2}$. The measurement of ultrasonic velocity in liquid mixtures is very useful and convenient tool to recognize the physicochemical behavior and molecular interactions. Knowledge of acoustical properties in solution furnishes a wealth of informations on molecular interactions occurring in the liquid mixtures, the nature and the strength of interactions. The temperature and concentration dependence of acoustic properties has proved to be a significant study of intermolecular interactions in liquids, liquid mixtures and solutions. Thus, the method of studying the molecular interactions from the knowledge of variation of acoustical parameters and their excess values with composition gives an insight into the molecular process ${ }^{3}$.

The investigations regarding the molecular interactions in ternary liquid mixtures having an alcohol as one component is of particular importance since alcohols are strongly self associated liquids with a three dimensional network of hydrogen bonds and can be 
associated with any other group having some degree of polar interactions ${ }^{4,5}$. Owing to these considerations, an attempt has been made to elucidate the molecular interactions in the mixtures of 1-propanol and 1-butanol with ethylmethacrylate in benzene at different temperatures.

\section{Experimental}

The chemicals used in the present work were analytical reagent and spectroscopic reagent grades with minimum assay of $99.9 \%$ were obtained from E-Merck Ltd. (India) and Sd Fine Chemicals, which were used as such without further purification. In both the systems, the mole fraction of the second component, ethyl methyl acetate $\left(\mathrm{X}_{2}=0.3\right)$ was kept constant, where the mole fractions of the remaining two were varied from 0.1 to 0.7 to have the mixtures of different compositions.

Ultrasonic velocity, density and viscosity measurements on liquid mixtures were made at three different temperatures 303,308 and $313 \pm 0.1 \mathrm{~K}$ respectively by using $\mathrm{F} 81$ Ultrasonic interferometer (Mittal Enterprises, New Delhi) at a frequency of $2 \mathrm{MHz}$ with an accuracy of $\pm 0.2 \%$, specific gravity bottle of capacity of $10 \mathrm{~mL}$ with accuracy of $\pm 0.0001 \mathrm{~g} \mathrm{~cm}^{-3}$ and the Ostwald's viscometer accurate to $\pm 0.1 \%$ respectively.

\section{Acoustical parameters}

In the present study, the ultrasonic velocity, density and viscosity measurements were carried out in the ternary mixtures of 1-propanol and 1-butanol with ethyl methacrylate in benzene at 303, 308 and $313 \mathrm{~K}$ at different concentrations.

Employing the measured values of velocity $(U)$, density $(\rho)$ and viscosity $(\eta)$, some acoustical parameters such as Adiabatic compressibility, Free length, Internal pressure, Free volume and their excess values can be computed through the following expression ${ }^{6,7}$.

$$
\begin{aligned}
\text { Adiabatic compressibility } & : \beta_{\mathrm{ad}}=\left[\mathrm{U}^{2} \rho\right]^{-1} \\
\text { Free length }: & \mathrm{L}_{\mathrm{f}}=\mathrm{K}\left[\beta_{\mathrm{ad}}\right]^{1 / 2} \\
\text { Free volume }: & \mathrm{V}_{\mathrm{f}}=\left[\mathrm{M}_{\mathrm{eff}} \mathrm{U} / \mathrm{K} \eta\right]^{3 / 2} \\
\text { Internal Pressure : } & \pi_{\mathrm{i}}=\mathrm{bRT}(\mathrm{K} \eta / \mathrm{u})^{1 / 2}\left(\rho^{2 / 3} / \mathrm{M}_{\mathrm{eff}}{ }^{7 / 6}\right)
\end{aligned}
$$

Where, $\mathrm{b}$ is a constant, which takes 2 for cubic packing, $\mathrm{R}$ is the gas constant, $\mathrm{K}$ is a temperature independent constant which is equal to $4.28 \times 10^{9}$ for all liquids.

\section{Excess parameters}

The study on the possible change of thermodynamic properties of mixtures and their degree of deviation from ideality is an excellent qualitative and quantitative way to investigate about the molecular structure and intermolecular forces in liquid mixtures. This has given impetus to the theoretical and experimental evaluation of the excess thermodynamic properties of liquid mixtures. Excess properties are sensitive towards external factors such as composition and temperature ${ }^{8}$.

The excess parameters have been evaluated using the following relation,

$$
\mathrm{Y}^{\mathrm{E}}=\mathrm{Y}_{\text {mix }}-\left[\mathrm{X}_{1} \mathrm{Y}_{1}+\mathrm{X}_{2} \mathrm{Y}_{2}+\mathrm{Y}_{3}\right]
$$

Where $\mathrm{Y}^{\mathrm{E}}$ is $\beta^{\mathrm{E}}$ or $\mathrm{L}_{\mathrm{f}}^{\mathrm{E}}$ or $\mathrm{V}_{\mathrm{f}}^{\mathrm{E}}$ or $\pi_{\mathrm{i}}^{\mathrm{E}}$ and $\mathrm{X}$ represent mole fraction of the component and subscript1, 2 and 3 for the components 1, 2 and 3 .

\section{Results and Discussion}

In the present investigation, different acoustical parameters such as ultrasonic velocity, adiabatic compressibility, intermolecular free length, free volume and internal pressure are calculated and listed in Table $1 \& 2$. The excess properties of the mixtures are listed in Table 3. 
It can be seen from the Table 1 that the ultrasonic velocity increases with increase in mole fraction of alcohols for all the mixtures, which indicates the presence of molecular association between solute and solvent. The structural changes of molecules in the mixture take place due to the existence of electrostatic field between the interacting molecules. Thus the structural arrangement of molecules results in the effect of adiabatic compressibility $(\beta)$. Adiabatic compressibility decreases with respect to the concentration in all the system since the molecules become more compact and less compressible as the new entities are formed. Such a continuous decrease in adiabatic compressibility with solute concentration has been qualitatively ascribed to the effect of hydrogen bonding'

Table 1. Measured values of ultrasonic velocity, density and viscosity of $n$-alkanols with ethylmethacrylate in benzene

\begin{tabular}{ccccccccccc}
\hline \multirow{2}{*}{ Mole Fraction } & \multicolumn{3}{c}{$\mathrm{U} \mathrm{ms}^{-1}$} & \multicolumn{3}{c}{$\rho \mathrm{kgm}^{-3}$} & \multicolumn{3}{c}{$\eta \times 10^{3} \mathrm{Nsm}^{-2}$} \\
\cline { 2 - 11 } & \multicolumn{3}{c}{ Temperature, $\mathrm{K}$} & \multicolumn{3}{c}{ Temperature, $\mathrm{K}$} & \multicolumn{4}{c}{ Temperature, $\mathrm{K}$} \\
\hline $\mathrm{X}_{1}$ & $\mathrm{X}_{3}$ & 303 & 308 & 313 & 303 & 308 & 313 & 303 & 308 & 313 \\
\hline \multicolumn{4}{c}{ System I 1-propanol } & with ethyl methacrylate in benzene \\
\hline 0.1000 & 0.6000 & 1094.7 & 1082.6 & 1071.8 & 934.39 & 933.59 & 932.34 & 0.7853 & 0.7408 & 0.6510 \\
0.1999 & 0.5000 & 1115.5 & 1106.7 & 1099.5 & 926.82 & 925.14 & 922.89 & 0.8023 & 0.7493 & 0.6849 \\
0.3000 & 0.4000 & 1133.2 & 1120.4 & 1109.6 & 916.08 & 914.20 & 913.10 & 0.8323 & 0.8067 & 0.7310 \\
0.4000 & 0.3000 & 1155.4 & 1146.9 & 1132.1 & 907.96 & 906.89 & 904.95 & 0.8868 & 0.8701 & 0.7618 \\
0.5000 & 0.2000 & 1176.7 & 1164.8 & 1150.4 & 893.17 & 893.05 & 890.41 & 0.9634 & 0.8926 & 0.8131 \\
0.6000 & 0.0999 & 1195.1 & 1183.3 & 1176.8 & 881.51 & 876.62 & 875.86 & 1.0674 & 1.0058 & 0.8896 \\
0.7000 & 0.0000 & 1219.3 & 1204.5 & 1195.3 & 868.50 & 865.34 & 863.39 & 1.2714 & 1.1786 & 1.1016 \\
\hline \multicolumn{1}{c}{ System II 1-butanol with ethyl methacrylate in benzene } \\
\hline 0.1000 & 0.6000 & 1109.7 & 1098.5 & 1085.4 & 932.8 & 931.0 & 930.7 & 0.7875 & 0.7645 & 0.6802 \\
0.2000 & 0.5000 & 1127.9 & 1118.2 & 1104.3 & 921.1 & 918.3 & 917.7 & 0.8183 & 0.7783 & 0.6896 \\
0.3000 & 0.4000 & 1146.2 & 1135.4 & 1127.6 & 910.2 & 909.6 & 905.6 & 0.9074 & 0.8497 & 0.7577 \\
0.4000 & 0.3000 & 1163.9 & 1158.1 & 1141.9 & 901.3 & 900.7 & 898.6 & 0.9864 & 0.9605 & 0.8176 \\
0.5000 & 0.1999 & 1184.5 & 1175.3 & 1159.7 & 890.0 & 888.0 & 881.1 & 1.1057 & 1.0445 & 0.9062 \\
0.6000 & 0.1000 & 1207.7 & 1194.5 & 1183.2 & 879.0 & 876.4 & 872.7 & 1.2906 & 1.1612 & 1.0512 \\
0.7000 & 0.0000 & 1226.3 & 1213.7 & 1208.5 & 866.6 & 864.1 & 862.0 & 1.5887 & 1.4635 & 1.2531 \\
\hline
\end{tabular}

Intermolecular free length is found to be a predominating factor, which depends upon adiabatic compressibility and shows a similar behavior as that of compressibility the addition of 1-ols with the mixture leads to a compact structure due to the presence of dipolar interaction this contributes to a decrease in free length and hence compressibility. The regular fall in free length with the mole fraction of alcohols may be attributed to the close approach of the molecules ${ }^{10}$. However, the increase in temperature makes the free length to increase due to the thermal expansion of liquid.

It is noticed that as the concentration of alcohols increases, free volume decreases where as the internal pressure increases. Due to this close packing of the molecules inside the shield takes place. The increase in internal pressure generally indicates association through hydrogen bonding and hence it supports the present investigation ${ }^{11}$ However, with rise in temperature, increase in free volume and decrease in internal pressure are noticed in all the system.

In order to understand more about the nature of interaction between the components of the liquid mixtures, it is necessary to discuss the same in terms of excess parameters rather than actual values. They can yield an idea about the non-ideality of the system as association or other types of interactions ${ }^{12,13}$. 
Table 2. Calculated values of acoustical parameters of $n$-alkanols with ethylmethacrylate in benzene

\begin{tabular}{|c|c|c|c|c|c|c|c|c|c|c|c|c|c|}
\hline \multicolumn{2}{|c|}{ Mole Fraction } & \multicolumn{3}{|c|}{$\begin{array}{c}\beta_{\text {ad }} \times 10{ }^{10} \mathrm{~Pa}^{-1} \\
\text { Temperature, } \mathrm{K}\end{array}$} & \multicolumn{3}{|c|}{$\begin{array}{c}\mathrm{L}_{\mathrm{f}} \times 10^{10} \mathrm{~m} \\
\text { Temperature, } \mathrm{K}\end{array}$} & \multicolumn{3}{|c|}{$\begin{array}{l}\mathrm{V}_{\mathrm{f}} \times 10^{7} \mathrm{~m}^{3} \mathrm{~mol}^{-1} \\
\text { Temperature, } \mathrm{K}\end{array}$} & \multicolumn{3}{|c|}{$\begin{array}{c}\pi_{\mathrm{i}} \times 10^{-6} \mathrm{~Pa} \\
\text { Temperature, } \mathrm{K}\end{array}$} \\
\hline $\mathrm{X}_{1}$ & $\mathrm{X}_{3}$ & 303 & 308 & 313 & 303 & 308 & 313 & 303 & 308 & 313 & 303 & 308 & 313 \\
\hline \multicolumn{14}{|c|}{ System I 1-Propanol with ethyl methacrylate in benzene } \\
\hline 0.1000 & 0.6000 & 8.930 & 9.1391 & 9.3367 & 5.6969 & 6.0801 & 6.2035 & 1.8012 & 1.9333 & 2.311 & 401.35 & 398.23 & 380.94 \\
\hline 999 & 0.5000 & 670 & & & & & & & & & 427.06 & & 42 \\
\hline 3000 & 0.4000 & 8.500 & & & & & & & & & 459.37 & & .45 \\
\hline 0.4000 & 0.3000 & 8.250 & & & & & & & & & 503.02 & 507 & .50 \\
\hline 0.5000 & 0.2000 & 8.086 & & & & & & & & & .58 & & .10 \\
\hline 000 & 0.0999 & 7.942 & & & & & & & & & 627.81 & & .09 \\
\hline 0.7000 & 0.0000 & 7.744 & 7.9652 & 8.1065 & 5.5592 & 5.6762 & 5.7803 & 0.5623 & 0.6185 & 0.676 & 736.74 & 723.71 & 712.71 \\
\hline \multicolumn{14}{|c|}{ System II 1-Butanol with ethyl methacrylate in benzene } \\
\hline 0.1000 & & 8.704 & 839010 & & & & & & & & & & 80.16 \\
\hline 1999 & 0.5000 & 8.533 & 8.7085 & 8.93 & & & & 1.7007 & 1.80 & 2.129 & 412.57 & & 394.41 \\
\hline 0.3000 & 0.4000 & 8.362 & 8.5273 & 8.6839 & 0.5776 & & 0.5982 & 1.3992 & 1.5222 & 1.789 & 449.47 & 444.05 & 426.36 \\
\hline 0.4000 & 0.3000 & 8.189 & 8.3926 & 8.5342 & 0.5716 & 0.5826 & 0.5930 & 1.1811 & 1.2074 & 1.520 & 486.83 & 491.04 & 461.31 \\
\hline 0.5000 & 0.2000 & 8.008 & 8.1516 & 8.4384 & 0.5652 & 0.5742 & 0.5897 & 0.9522 & 1.0251 & 1.243 & 535.15 & 530.00 & 502.41 \\
\hline 6000 & 0.0999 & 7.799 & 7.9966 & 8.1843 & 0.5578 & 0.5687 & 0.5808 & 0.7221 & 0.8323 & 0.952 & 601.43 & 581.91 & 563.76 \\
\hline 0.7000 & 0.0000 & 73672 & 7.8559 & 7.9423 & 0.5533 & 0.5637 & 0.5721 & 0.5005 & 0.5574 & 0.699 & 696.79 & 682.00 & 641.69 \\
\hline
\end{tabular}


Table 3. Excess values of Acoustical parameters of $n$-alkanols with ethylmethacrylate in benzene

\begin{tabular}{|c|c|c|c|c|c|c|c|c|c|c|c|c|c|}
\hline \multicolumn{2}{|c|}{ Mole Fraction } & \multicolumn{3}{|c|}{$\begin{array}{l}\beta^{\mathrm{E}} \times 10^{10} \mathrm{~Pa}^{-1} \\
\text { Temperature, } \mathrm{K}\end{array}$} & \multicolumn{3}{|c|}{$\begin{array}{c}\mathrm{L}_{\mathrm{f}}^{\mathrm{E}} \times 10^{10} \mathrm{~m} \\
\text { Temperature, } \mathrm{K}\end{array}$} & \multicolumn{3}{|c|}{$\begin{array}{l}\mathrm{V}_{\mathrm{f}}^{\mathrm{E}} \times 10^{7} \mathrm{~m}^{3} \mathrm{~mol}^{-1} \\
\text { Temperature, } \mathrm{K}\end{array}$} & \multicolumn{3}{|c|}{$\begin{array}{c}\pi_{\mathrm{i}}^{\mathrm{E}} \times 10^{-6} \mathrm{~Pa} \\
\text { Temperature, } \mathrm{K}\end{array}$} \\
\hline $\mathrm{X}_{1}$ & $\mathrm{X}_{3}$ & 303 & 308 & 313 & 303 & 308 & 313 & 303 & 308 & 313 & 303 & 308 & 313 \\
\hline \multicolumn{14}{|c|}{ System I 1-Propanol with ethyl methacrylate in benzene } \\
\hline 0.1000 & 0.6000 & 0.9367 & 0.8881 & 0.8521 & 0.0326 & 0.0284 & 0.0294 & -1.8165 & -2.100 & -1.888 & -350.7 & -342.7 & -336.4 \\
\hline 0.1999 & 0.5000 & 0.6289 & 0.5243 & 0.4220 & 0.0222 & 0.0139 & 0.0149 & -1.5327 & -1.7399 & -1.6663 & -415.1 & -404.9 & -394.7 \\
\hline 0.3000 & 0.4000 & 0.4105 & 0.3628 & 0.2977 & 0.0148 & 0.0062 & 0.0107 & -1.2844 & -1.5510 & -1.5028 & -479.4 & -467.1 & -453.0 \\
\hline 0.4000 & 0.3000 & 0.1119 & -0.0179 & -0.0317 & 0.0045 & -0.0090 & -0.0005 & -1.0656 & -1.3020 & -1.2188 & -543.8 & -529.3 & -511.4 \\
\hline 0.5000 & 0.2000 & -0.0996 & -0.1967 & -0.2229 & -0.0027 & -0.0174 & -0.0070 & -0.8523 & -0.9518 & -0.9720 & -608.2 & -591.5 & -569.7 \\
\hline 0.6000 & 0.0999 & -0.2920 & -0.3537 & -0.5219 & -0.0095 & -0.0251 & -0.0174 & -0.6330 & -0.7245 & -0.7233 & -672.6 & -653.7 & -628.0 \\
\hline 0.7000 & 0.0000 & -0.5380 & -0.5854 & -0.7161 & 0.0821 & -0.0355 & -0.0242 & -0.4324 & -0.4766 & -0.5824 & -736.9 & -715.9 & -686.3 \\
\hline \multicolumn{14}{|c|}{ System II 1-Butanol with ethyl methacrylate in benzene } \\
\hline 0.1000 & 0.6000 & 0.7726 & 0.7056 & 0.6994 & 0.0269 & 0.0246 & 0.0243 & -1.7449 & -2.1064 & -1.9436 & -341.8 & -332.7 & -327.9 \\
\hline 0.1999 & 0.5000 & 0.6147 & 0.5189 & 0.5234 & 0.0213 & 0.0183 & 0.0184 & -1.4743 & -1.7308 & -1.5735 & -397.1 & -384.8 & -377.8 \\
\hline 0.3000 & 0.4000 & 0.4558 & 0.3418 & 0.2786 & 0.0155 & 0.0122 & 0.0100 & -1.3360 & -1.5274 & -1.4206 & -452.7 & -437.1 & -427.8 \\
\hline 0.4000 & 0.3000 & 0.2960 & 0.2121 & 0.1363 & 0.0097 & 0.0077 & 0.0051 & -1.1144 & -1.3511 & -1.1952 & -508.1 & -489.3 & -477.7 \\
\hline 0.5000 & 0.2000 & 0.1271 & -0.0239 & 0.0479 & 0.0035 & -0.0005 & 0.0020 & -0.9035 & -1.0422 & -0.9791 & -563.6 & -541.5 & -527.7 \\
\hline 0.6000 & 0.0999 & -0.0686 & -0.1738 & 0.1985 & -0.0037 & -0.0058 & -0.0066 & -0.6936 & -0.7436 & -0.7758 & -619.1 & -593.7 & -577.6 \\
\hline 0.7000 & 0.0000 & -0.1823 & -0.3097 & -0.4333 & -0.0081 & -0.0107 & -0.0150 & -0.4756 & -0.5276 & -0.5360 & -674.5 & -645.9 & -627.5 \\
\hline
\end{tabular}


The variation of excess adiabatic compressibility, excess free length, excess free volume and excess internal pressure against the composition of alcohols in all the ternary systems at different temperatures are shown in Table 3. It can be observed that the excess compressibility is positive at lower mole fraction and it becomes negative at higher mole fraction in both 1-propanol and 1-butanol system. The negative excess compressibility at higher mole fraction may be due to close packed molecules while positive excess values are due to weak interaction between unlike molecules. Anwar Ali et. al., ${ }^{14}$ suggested that the negative excess values of compressibility indicate the formation of hydrogen bond between unlike molecules the values of excess intermolecular free length $\mathrm{L}_{\mathrm{f}}{ }^{\mathrm{E}}$ follow the same trend as that of $\beta^{\mathrm{E}}$. The values of excess free volume are negative in all the system. The negative deviation of excess free volume is an indication of the existence of strong interaction between the components.

\section{Conclusion}

The variation of ultrasonic velocity, calculated acoustical parameters and magnitude of the excess functions suggest that the presence of molecular interaction in the ternary mixtures.

- Molecular interaction is due to the formation of hydrogen bonding through polar oxygen of EMA and hydrogen atom of alcohols.

- The observed negative excess values in all the mixture indicate the strong interactions between the unlike molecules.

- The temperature dependence of the different parameters suggests that the degree of deviation from ideality is temperature sensitive and influenced by the strong hydrogen bonds.

\section{References}

1. Pimental G C and Maclellan A L, The Hydrogen Bond, (W H Freeman and Co., San Fransisco), 1960.

2. Mistry A A, Bhandakkar V D and Chiankar O P, J Chem Pharm Res., 2012, 4(1), 170-174.

3. Kincaid J F and Eyring H, J Chem Phys., 1937, 5, 587.

4. Rowlison J S, Liquid and Liquid Mixtures ( $2^{\text {nd }}$ Edn.), London: Butter Worths, 1969, 159.

5. Ali A, Nain A K and Abida, J Chem Soc., 2004, 51, 477-485.

6. $\quad$ Verma H S and Vinod Kumar, Int J Pure Appl Chem., 2007, 2(4), 387.

7. Kumar R, Jayakumar S and Kannappan V, Indian J Pure Appl Phys., 2008, 46, 169-175.

8. Praveen S, Yasmin M, Gupta M and Shukla J P, Int J Thermodynamics, 2010, 13(2), 59-66; DOI:10.5541/ijot.1034000282

9. $\quad$ Ali A and Soghra H, Indian J Phys., 2002, 76B(1), 23.

10. Niham P S, Kapade V M and Mehdi Hasan, Indian J Pure Appl Phys., 2000, 38, 170-173.

11. Kannappan V and Hemalatha G, Indian J Pure Appl Phys., 2005, 43, 849-853.

12. Surjit Singh Batti and Devinder Pal Singh, Indian J Pure Appl Phys., 1983, 21, 506.

13. Kannappan A N and Palaniappan L, Indian J Phys., 1999, 73(B), 531.

14. Anwar Ali, Anil Kumar Nain, Vinod Kumar Sharma and Shakil Ahmad, Indian $J$ Pure Appl Phys., 2004, 42, 666. 